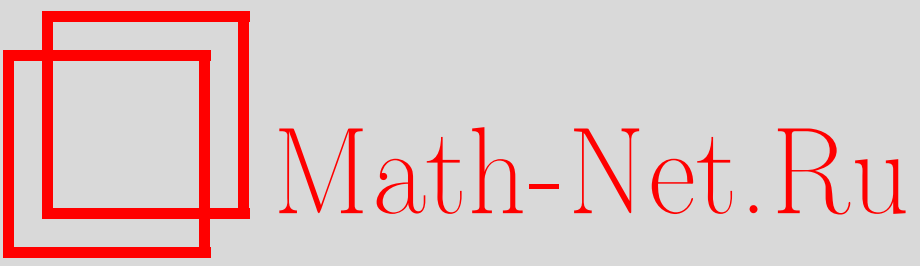

М. В. Шамолин, О грубости диссипативных систем и относительной грубости и негрубости систем с переменной диссипацией, УМН, 1999, том 54, выпуск 5, 181-182

DOI: https://doi.org/10.4213/rm217

Использование Общероссийского математического портала Math-Net.Ru подразумевает, что вы прочитали и согласны с пользовательским соглашением

http://www. mathnet.ru/rus/agreement

Параметры загрузки :

IP: 54.89 .56 .158

26 апреля 2023 г., 18:34:01 


\title{
О ГРУБОСТИ ДИССИПАТИВНЫХ СИСТЕМ И ОТНОСИТЕЛЬНОЙ ГРУБОСТИ И НЕГРУБОСТИ СИСТЕМ С ПЕРЕМЕННОЙ ДИССИПАЦИЕЙ
}

\author{
М. В. ШАмолин
}

В работе приводится краткий обзор вопросов относительной структурной устойчивости (относительной грубости) динамических систем, рассматриваемых не на всем пространстве динамических систем, а лиш на некотором его подпространстве [1].

Действительно, в ряде вопросов представляет интерес рассмотрение грубости по отношению к некоторому классу динамических систем. Таким понятием относительной грубости для начала можно воспользоваться при выделении простейших негрубых систем, т.е. систем первой степени негрубости, а также при классификации негрубых систем по степени сложности или степени негрубости.

Поэтому, рассматривая класс консервативных систем, можно ввести понятие грубости системы относительн этого класса [1]. Таким понятием (без термина “грубость”) пользовался Пуанкаре [2].

В силу определения систем первой степени негрубости [3] они являются относительно грубыми в множестве негрубых систем.

Определение, данное Андроновым и Портнягиным [4], наряду с близостью в некоторой топологии рассматриваемой системы и ее деформации требует близость к тождественному гомеоморфизма, через который осуществляется топологическая эквивалентность. Определение же, данное Пейшото [5], не требует указанной близости.

В высших размерностях множество грубых полей по-прежнему обширно, но не является уже всюду плотным. Здесь существуют богатые и более сложные явления, сохраняющиеся при малых возмущениях первоначального поля.

За последнее время появилось несколько видоизмененных определений грубости. Все они имеют одно общее сходство: деформация рассматриваемых динамических систем на многообразии $M^{n}$ берется во всем пространстве гладких векторных полей $\chi\left(C^{r}\right)$ в $C^{r}$-топологии.

Поэтому важно рассматривать векторные поля (динамические системы), деформируемые не над всем классом $\chi\left(C^{r}\right)$ полей, а лишь над некоторым подклассом $\chi(\mathscr{B})$, определенным с помощью класса функций $\mathscr{B} \subset C^{r}$. Подобно тому, как дается определение векторного поля первой степени негрубости, можно определить поля первой степени относительной негрубости, рассматривая деформации полей в подпространстве $\chi(\mathscr{B})$ пространства всех векторных полей [1].

1. Системы с переменной диссипацией в плоской динамике твердого тела. Такие системы обладают одним общим свойством: поскольку, как правило, у систем, обладающих переменной диссипацией, существуют дополнительные симметрии, данные системы имеют сепаратрисы, соединяющие гиперболические седловые положения равновесия. Поэтому (абсолютно) структурно устойчивыми такие системы быть не могут.

Поскольку деформации таких систем рассматриваются лишь над некоторым подмножеством всех систем, определенным с помощью подкласса функций (правых частей), позволяющего сохранить все симметрии в системе, рассматриваемые системы в некоторых областях параметров остаются относительно грубыми [1].

Из всего вышеизложенного видно, что чисто диссипативные системы [6], [7] в типичном случае скорее всего (абсолютно) структурно устойчивы. В общем случае данный факт нужно доказывать. При этом относительно структурно устойчивые (типичные) системы с переменной диссипацией являются, как правило, удобными системами сравнения [8], [9] для чисто диссипативных систем.

2. Системы с переменной диссипацией и диссипативные системы в пространственной динамике твердого тела. Аналогичные рассуждения справедливы и для систем, 
возникающих в пространственной динамике твердого тела. При этом системы с переменной диссипацией являются лишь относительно грубыми, а чисто диссипативные системы - просто грубыми. Такие системы являются нетривиальными примерами относительно и абсолютно грубых систем, имеюших порядок выше двух [10].

Системы с переменной диссипацией возникают естественным образом в пространственной динамике твердого тела благодаря наличию дополнительных групп симметрий. Последние возникают из естественных (геометрических и аналитических) симметрий задачи. Таким образом, наряду с "полезностью" возникновения систем с переменной диссипацией в качестве важных систем сравнения они имеют право на существование ввиду их относительной грубости. А вот чисто диссипативные системы уже по своей физической и топологической природе, как правило, являются (абсолютно) грубыми, что позволяет говорить о структурно устойчивом описании природных явлений с помощью таких систем. Например, системы вида ( $\sigma, B, m$ - постоянные)

$$
\begin{gathered}
\alpha^{\prime}=-Z_{2}+\sigma\left(Z_{1}^{2}+Z_{2}^{2}\right) \sin \alpha+\frac{\sigma}{B} \mathscr{F}(\alpha) \cos \alpha+\frac{s(\alpha)}{m} \sin \alpha, \\
Z_{2}^{\prime}=\frac{1}{B} \mathscr{F}(\alpha)-Z_{2} \Psi_{1}\left(\alpha, Z_{1}, Z_{2}\right)-Z_{1}^{2} \frac{\cos \alpha}{\sin \alpha}, \\
Z_{1}^{\prime}=-Z_{1} \Psi_{1}\left(\alpha, Z_{1}, Z_{2}\right)+Z_{1} Z_{2} \frac{\cos \alpha}{\sin \alpha}, \\
\Psi_{1}\left(\alpha, Z_{1}, Z_{2}\right)=-\sigma\left(Z_{1}^{2}+Z_{2}^{2}\right) \cos \alpha+\frac{\sigma}{B} \mathscr{F}(\alpha) \sin \alpha-\frac{s(\alpha)}{m} \cos \alpha,
\end{gathered}
$$

зависящие от двух классов функций $\{\mathscr{F}\}$ и $\{s\}$ (об их определении см. [1], [2], [8]) (абсолютно) грубы. При $s \equiv 0$ такие системы являются относительно грубыми системами с переменной диссипацией и не являются грубыми (абсолютно).

\section{СПИСОК ЛИТЕРАТУРЫ}

[1] Шамолин М.В.// УМН. 1996. Т. 51. № 1. С. 175-176. [2] Пуанкаре А. О кривых, определяемых дифференциальными уравнениями. М.-Л.: ОГИЗ, 1947. [3] Баутин Н. Н., Лефонтович Е. А. Методы и приемы качественного исследования динамических систем на плоскости. М.: Наука, 1976. [4] Андронов А. А., Понтрягин Л.С. // Докл. АН СССР. 1937. T. 14. № 5. C. 247-250. [5] Peixoto M. // Topology. 1962. V. 1. № 2. P. 101-120. [6] Шамолин М.В.// Докл. РАН. 1994. Т. 337. №5. С. 611-614. [7] Шамолин М. В. // Докл. РАН. 1996. Т. 349. №2. С. 193-197. [8] Шамолин М. В. // Вестн. МГУ. Матем., мех. 1993. № 2. С. 66-70, 113. [9] Шамолин М. В. // УМН. 1997. Т. 52. № 3. С. 177-178. [10] Шамолин М. В. // Изв. РАН. Мех. тверд. тела. 1997. № 2. С. 65-68. 